\title{
Systèmes D'information et Résilience De La Chaine Logistique Globale: Étude Exploratoire Auprès des Entreprises De L'industrie Automobile Au Maroc
}

\author{
Kamar Moukadem, Phd Student \\ Abdelilah Elkharraz, PH
}

Groupe de recherche: Management \& Systèmes d'information (GRMSI)

Ecole Nationale de Commerce et de Gestion de Tanger

Université Abdelmalek Essaadi, Maroc

Doi:10.19044/esj.2019.v15n34p367 URL:http://dx.doi.org/10.19044/esj.2019.v15n34p367

\section{Résumé}

L'objectif de cet article est d'expliquer le processus par lequel les systèmes d'information contribuent à la résilience de la chaine logistique globale. Sur le plan méthodologique, nous avons adopté une approche à caractère exploratoire en menant une étude qualitative auprès de sept entreprises opérant dans le secteur automobile au Maroc. Les résultats obtenus montrent que la contribution des systèmes d'information à la résilience de la chaine logistique est dépendante de plusieurs variables, parmi lesquelles les capacités du système d'information constituent une variable centrale permettant de faire face aux incertitudes des supply chains et d'accroître leurs résiliences. Les résultats montrent aussi que la résilience de la chaine logistique reste un concept méconnu et difficile à opérationnaliser pour la plupart des cas étudiés. En associant ce concept aux quatre capacités de la résilience, une définition synthétique de la résilience de la chaine logistique, adaptée au contexte de l'industrie automobile est proposée.

Mots clés: Systèmes d'Information, Résilience, Chaine Logistique, Département Logistique, Industrie Automobile, Maroc 


\title{
Information Systems and Global Supply Chain Resilience : An Exploratory Study of Companies for the Automotive Industry in Morocco
}

\author{
Kamar Moukadem, Phd Student \\ Abdelilah Elkharraz, $\mathbf{P H}$
}

Groupe de recherche: Management \& Systèmes d'information (GRMSI)

Ecole Nationale de Commerce et de Gestion de Tanger

Université Abdelmalek Essaadi, Maroc

\begin{abstract}
This article aims to explain the process by which information systems contribute to the global supply chain resilience. Methodologically, we adopted an exploratory approach by conducting a qualitative study of seven companies in the automotive industry in Morocco. The results obtained assert that the contribution of information systems to the resilience of the supply chain is dependent on several variables, among which the information system capabilities constitute a central variable to face the uncertainties of supply chains and increase their resilience. The results also show that the resilience of the supply chain remains an unknown concept that is difficult to operationalize for most of the cases studied. By combining this concept with the four capabilities of resilience, a synthetic definition of the resilience of the supply chain adapted to the context of the automotive industry is proposed.
\end{abstract}

Keywords: Information Systems, Resilience, Supply Chain, Logistics Department, Automotive Industry, Morocco

\section{Introduction}

Les changements récents dans le monde des affaires, les améliorations apportées au supply chain management ainsi que les changements dans la structure industrielle des pays en développement ont permis à ces pays d'intégrer les chaînes logistiques globales (COMTD, 1996). L'exemple typique de cette intégration est l'industrie automobile, où les réseaux de production et de distribution ont globalement émergé et où les fournisseurs des pays en développement prennent leur part (Fleury \& Fleury, 2001).

Au Maroc, l'industrie automobile n'échappe pas à cette réalité imposée par le marché. Cette dernière est caractérisée par une supply chain complexe 
et fragile (Al Meriouh, Y., Elkharraz, A., \& Al Barrak, F. 2017). Ceci s'explique principalement par les incertitudes auxquelles sont confrontés les partenaires de la supply chain (Wang et al., 2006) et par la dépendance des entreprises à l'égard de leurs partenaires de la chaine (Kumar et al., 1995). Une façon de gérer ces incertitudes consiste à conférer à la supply chain automobile la capacité d'être résiliente.

Ainsi, une supply chain résiliente doit développer des capacités afin de réagir aux conséquences négatives d'événements imprévus, de revenir rapidement à son état d'origine, ou de passer à un état optimal après avoir été perturbée (Pettit et al., 2010; Peck, 2005 ; Carvalho et al., 2012).

Pour ce faire, les entreprises de l'industrie automobile au Maroc ont tendance à investir massivement dans la mise en place des systèmes d'information inter-organisationnels avec leurs partenaires de la chaine logistique (Boubker \& Chafik, 2015, 2016, 2017). Ces systèmes favorisent l'intégration entre les acteurs de la chaine, rendant alors l'information disponible pour des prises de décisions réactives et par conséquent, ils contribuent à la résilience de la chaine logistique globale (Prajogo et Olhager, 2012; Ngai, Chau et Chan, 2011; Pan et al., 2012, Boubker, 2017).

Parce que chaque entreprise de l'industrie automobile est au cœur d'un réseau (Anderson, 1994) et liée par des processus logistiques interorganisationnels, cet article vise à étudier les liens entre l'usage des systèmes d'information qui les soutiennent et la résilience de la chaîne logistique globale. Ainsi, la problématique générale de la recherche peut s'énoncer comme suit : Dans quelle mesure l'usage des systèmes d'information contribue-t-il à la résilience de la chaine logistique globale ?

Afin d'apporter des éléments de réponse à notre problématique générale, des questions dérivées de recherche vont nous guider :

Quelles sont les variables à prendre en compte pour étudier la contribution des SI à la résilience de la chaine logistique ?

Quels sont les liens entre les variables prises en compte pour l'étude de la contribution des SI à la résilience de la chaine logistique ?

Evrard Samuel et Ruel (2013) notent que les travaux de recherche en matière de la modélisation de la résilience de la chaine logistique se concentrent davantage sur la rupture des flux physiques, qui est facilement observable et chiffrable, et pourtant peu de chercheurs ont essayé de comprendre en quoi les systèmes d'information, peuvent-ils contribuer à la capacité de résilience de leurs supply chains.

Cette étude s'inscrit dans cette problématique. L'objectif consiste à proposer un modèle explicatif de la contribution des SI à la résilience de la chaine logistique globale, à travers une étude qualitative exploratoire auprès de sept entreprises de l'industrie automobile au Maroc. Pour cela, nous commençons, dans la première section, par une synthèse de la littérature 
permettant de mettre l'accent sur la résilience de la chaine logistique d'une part, et ses liens avec les systèmes d'information d'une autre. Dans la deuxième section, nous présentons notre modèle théorique via la justification du choix du modèle de référence. Le cadre empirique de la recherche sera présenté dans une troisième section. Dans la quatrième section, nous présentons et discutons les principaux résultats obtenus. Enfin, nous discutons quelques limites et perspectives de notre recherche.

\section{Revue De Littérature}

\section{La résilience de la chaine logistique}

Avec la globalisation des supply chains, les relations inter-acteurs se caractérisent par un grand degré de complexité lié à la multiplicité des intervenants et à la longueur des chaines logistiques (Blackhurst, Dunn \& Craighead, 2011, Christopher \& Peck, 2004, Pettit, Croxton \& Fiksel, 2010). Un tel accroissement de la complexité a rendu les entreprises de l'industrie automobile plus que jamais vulnérables aux perturbations de la chaine logistique. Les conséquences peuvent avoir un effet direct sur la capacité à poursuivre les opérations et à servir les clients (Jüttner, 2005); d'où l'importance du concept de la résilience de la chaîne logistique, qui est devenu d'intérêt accru pour faire face à ces perturbations (Jüttner \& Maklan, 2011).

La résilience de la supply chain permet de réduire l'impact d'une perturbation en offrant la capacité de se rétablir rapidement (Sheffi \& Rice, 2005). Une chaîne logistique qui est encore capable d'exécuter et de fournir des produits et des services dans telles circonstances est caractérisée comme résiliente (Hohenstein et al., 2015).

Étant donné que la résilience est un domaine émergent dans la gestion de la chaîne logistique (SCM), il n'existe pas une définition généralement acceptée et communément utilisée pour cette approche multidisciplinaire et multidimensionnelle (Wieland et Wallenburg, 2013).

La revue de littérature révèle un manque de consensus clair et d'uniformité entre les chercheurs sur la définition de la résilience de la chaîne logistique. Certains chercheurs définissent la résilience de la chaîne logistique dans une approche réactive. Il s'agit d'une capacité de réaction et d'adaptation ou de résistance aux incidents imprévus (Pettit \& Fiksel, 2010; Rice \& Caniato, 2003; Wu, Huang, Blackhurst, Zhang, 2009 ; \& Wang, 2013). D'autres l'abordent dans une approche proactive. Ces derniers considèrent la résilience de la chaîne logistique comme une capacité à anticiper, à se préparer à l'avance et à éviter une perturbation (Klibi, Martel et Guitouni, 2010, Priya Datta, Christopher et Allen, 2007).

La plupart des recherches récentes examinent trois phases distinctes de la résilience: la préparation, la réponse et le rétablissement (Chowdhury et Quaddus, 2016). Pour comprendre la résilience dans le contexte de la supply 
chain, Ponomarov et Holcomb (2009), à travers une étude approfondie de la littérature dans différentes disciplines (psychologie, écosystème...), ont défini la résilience comme «la capacité adaptative des chaînes logistiques à se préparer à des évènements inattendus, à répondre aux perturbations et à s'en remettre tout en maintenant la continuité des opérations à un niveau souhaité de connexion et de contrôle sur la structure et ses fonctions». Ainsi, pour qu'une chaîne logistique soit résiliente, les partenaires de la supply chain doivent développer des capacités d'anticipation, de réponse et de rétablissement afin de réduire la vulnérabilité de leurs chaines logistiques aux perturbations (Ponomarov, 2012). Pour ce faire, la revue de littérature met en évidence plusieurs stratégies définies, par certains auteurs, comme des éléments formatifs de la résilience (Blackhurst et al., 2011; Hohenstein et al., 2015). D’autres les considèrent comme antécédents de la résilience de la supply chain (Ponomarov et Holcomb, 2009); des concepts tels que (l'intégration, la visibilité, la collaboration, la redondance, la flexibilité, l'agilité et l'adaptabilité) ne sont que quelques antécédents de la résilience communément discutés dans la littérature.

Malgré qu'un large éventail de différentes capacités soit employé dans la littérature pour le contexte d'une gestion résiliente, celles-ci convergent vers deux principales capacités ou antécédents, considérées comme ayant une plus grande influence sur la résilience de la supply chain à savoir : 1'intégration et la flexibilité (Xavier Brusset \& Christoph Teller, 2016).

\section{Les systèmes d'information et la résilience de la chaine logistique}

L'enjeu de la chaîne logistique globale, reliant différentes unités (intra ou interentreprises), est d'assurer la continuité et la fluidité des flux physiques entre les différentes unités par une exploitation globalement optimisée des flux informationnels. Ce qui permet par conséquent d'assurer une coordination entre l'ensemble des entreprises de la chaine logistique. Cette coordination ne peut être sans une mise en place des systèmes d'information, permettant aux acteurs de la supply chain d'échanger, de partager ou de mettre à disposition les informations tout au long de la chaine logistique globale (Chen, 2013).

La littérature en management des systèmes d'information permet de repérer deux principaux niveaux d'intégration des processus, ayant pour objectif de soutenir la coordination au sein de la chaîne logistique : le niveau intra-organisationnel et le niveau inter-organisationnel (Markus, 2001).

Le premier niveau a pour objectif de soutenir les processus interfonctionnels au sein de la même organisation (Corbière et al., 2012). Le second niveau tend à améliorer la collaboration entre les différentes organisations contribuant à la même chaîne logistique à travers la mise en place des systèmes automatisés partagés qualifiés des SIIO «Systèmes d'Informations InterOrganisationnels» (Wei et al., 2012). 
Un système d'information inter-organisationnel (SIIO) a été défini comme un système permettant l'échange et le partage des informations par une ou plusieurs organisations, en créant des ressources d'information communes telles que les bases de données, les logiciels ou les réseaux de communication (Aubert et Dussart, 2002).

Par ailleurs, le marché d'aujourd'hui est relié électroniquement et de nature dynamique. Plus une entreprise met en réseau ses systèmes d'information, plus elle est menacée qu'une erreur quelque part peut causer des erreurs partout (Chopra \& Sodhi, 2004). Par conséquent, les entreprises essaient d'améliorer la résilience de leurs supply chains dans le but d'être flexible et réactive pour répondre aux différentes perturbations.

Le lien entre systèmes d'information et résilience logistique a fait l'objet d'un nombre restreint de publications académiques (Evrard Samuel et Ruel, 2013).

Dans un environnement incertain, plusieurs chercheurs ont postulé que le partage de l'information et de la connaissance via un système d'information, entre les partenaires de la chaîne logistique, réduit l'incertitude et facilite des réactions et des interventions utiles ainsi qu'une prise des décisions efficaces (Mentzer et al., 2001; Harrison et van Hoek, 2005). Dans la même lignée d'idée, Evrard Samuel et Ruel (2013, P.78) expliquent qu' « en cas de rupture brutale des flux, le SI devrait être en mesure de fournir les informations nécessaires afin de rétablir et stabiliser les flux d'information. Les technologies actuellement disponibles et en particulier les outils du Web 2.0 permettent de créer du lien, d'encourager les interactivités et de favoriser les connexions entre l'ensemble des maillons d'une supply chain ».

Ainsi, grâce au partage et au transfert d'informations en temps réel entre les différents acteurs de la chaine logistique et sa capacité décisionnelle, le système d'information permet d'anticiper, de réagir et de s'adapter à un environnement changeant, et par conséquent de contribuer à la résilience de la chaine logistique globale (Ouabouch et Paché, 2014).

\section{Modele Theorique De Recherche}

Dans le panorama de la recherche sur l'évaluation des systèmes d'information inter-organisationnels, la notion d'alignement (FIT) est saillante (Venkatraman, 1989). Les recherches qui reposent sur le construit du fit ont pour objet d'évaluer l'adéquation des capacités de la technologie adoptée aux besoins de l'organisation, afin d'atteindre les objectifs de la performance visés par l'utilisation des systèmes d'information (Bostrom, et Heinen, 1977). Dans ce contexte, Bensaou et Venkatraman (1995) ont proposé, un modèle conceptuel de coordination inter-organisationnelle dans le cadre des relations inter-organisationnelles liées aux technologies de l'information. En se servant de ce modèle et des travaux de recherche traitants 
la modélisation de la résilience de la chaine logistique et des systèmes d'information, Elkharraz et Moukadem (2019) ont élaboré un nouveau modèle théorique permettant l'évaluation de la contribution des SI à la résilience de la chaine logistique globale (Figure 1).

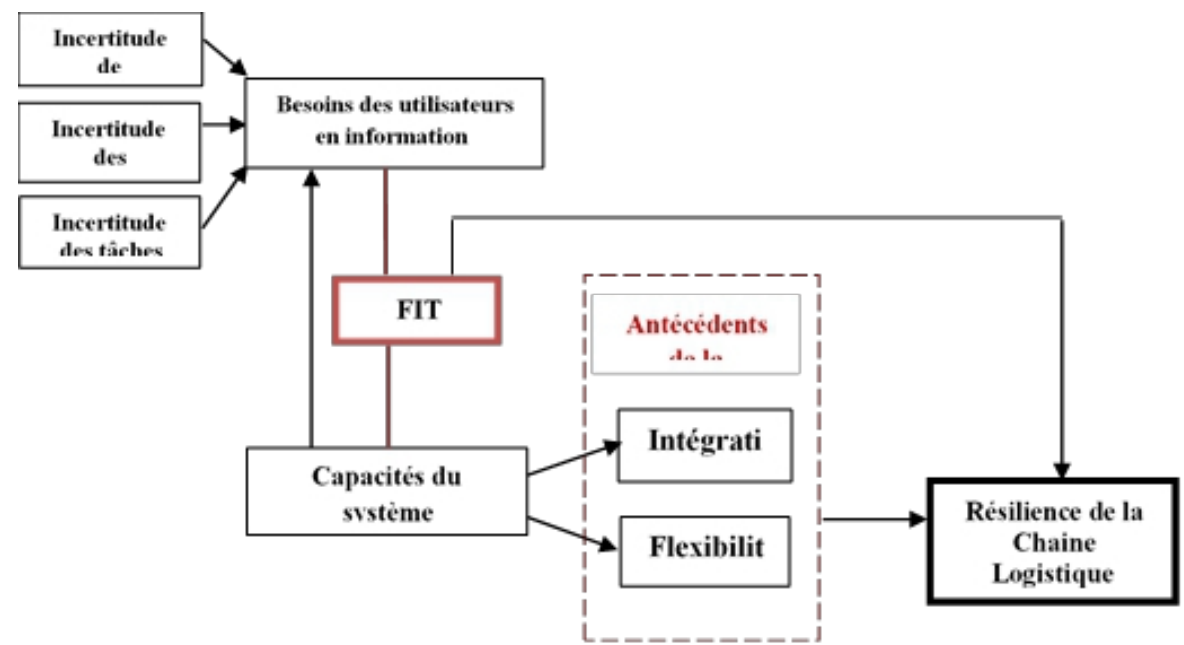

Figure 1 : Modèle théorique de recherche (Elkharraz et Moukadem, 2019, p.729)

\section{Cadre Methodologique}

\section{Le Choix De La Méthode De L'étude De Cas}

La raison du choix d'une méthode qualitative est le caractère exploratoire de la recherche (Yin, 1994; Eisenhardt, 1989). La méthode qualitative est identifiée comme étant apte à favoriser une profonde compréhension des interprétations des acteurs étudiés (Pratt, 2009).

Nous choisissons l'étude de cas comme stratégie de recherche empirique qui étudie un phénomène contemporain dans un contexte réel, lorsque les frontières entre le phénomène et le contexte n'apparaissent pas clairement, et dans laquelle on mobilise des sources empiriques multiples (Yin, 2009).

L'étude de cas est appropriée aux recherches qui s'interrogent sur le « comment » d'un ensemble contemporain d'évènements (Yin, 2009). Elle est pertinente car elle permet de mettre en lumière et d'illustrer, un phénomène jusqu'alors peu accessible (Thiétart et al., 2007) ; ce qui convient parfaitement à la thématique de la résilience de la supply chain qui reste un nouveau domaine peu abordé par la littérature du supply chain management (Ponomarov, 2012).

A ce stade, nous confirmons la pertinence de l'étude de cas pour notre problématique de recherche, qui nécessite des études en profondeur dans la mesure où nous cherchons à comprendre les liens entre les SI et la capacité 
résiliente de la supply chain des entreprises de l'industrie automobile face aux différentes incertitudes de leurs chaînes logistiques.

\section{Terrain d'investigation et profil des acteurs interrogés}

Nous avons choisi d'étudier la chaîne logistique des entreprises de l'industrie automobile, cependant, il nous faut également choisir le nombre de cas adéquat à notre recherche. Dans la littérature, il n'y a pas un consens sur le nombre idéal des cas à utiliser dans une recherche basée sur des cas multiples. C'est pour cette raison que nous nous sommes basés sur le principe de saturation théorique, qui est atteinte lorsqu'on ne trouve plus de données supplémentaires, générant de nouveaux éclairages théoriques, ni de nouvelles propriétés aux principales catégories (Charmaz, 2006).

Ainsi, dans notre étude, le seuil de saturation théorique a été atteint au niveau de la sixième entreprise de l'industrie automobile, à partir de laquelle nous ne récoltions plus de nouvelles informations. Sur la base des réponses récoltées auprès de ces six entreprises, nous avons jugé très intéressant d'ajouter à notre échantillon la perception d'un prestataire du service logistique à destination d'industriels automobiles, vu le rôle déterminant et la place de plus en plus importante qu'il joue au sein de la supply chain (Boissinot, 2010), et en tant qu'acteur incontournable de l'industrie automobile, dans la mesure où il peut s'intégrer en amont et en aval de la chaine logistique, et ce dans le but d'apporter un éclairage empirique aux incertitudes liées à l'import et à l'export, et de consolider l'analyse des résultats obtenus auprès des six entreprises de l'industrie automobile.

Les informations nécessaires pour cette recherche sont détenues à travers des entretiens individuels en face à face, auprès des logisticiens et décideurs participant activement aux processus logistiques. Néanmoins, quelques uns de nos interviewés ne participent pas directement à l'activité logistique mais font partie du service support lié au système d'information dédié à la logistique (Département IT). Nous avons jugé important d'intégrer leur perception sur l'usage du système d'information logistique.

Le tableau ci-après donne un descriptif synthétique des entreprises et des interlocuteurs rencontrés avec leurs principales tâches au sein de la supply chain automobile. 
Tableau 1: Présentation de l'échantillon de l'étude exploratoire

\begin{tabular}{|c|c|c|c|}
\hline Entreprise & Activité & $\begin{array}{l}\text { Personne } \\
\text { interviewée }\end{array}$ & Principales tâches \\
\hline \multirow[t]{2}{*}{ A } & \multirow[t]{2}{*}{$\begin{array}{l}\text { Constructeur } \\
\text { automobile }\end{array}$} & Responsable achat & $\begin{array}{l}\text { Choix des fournisseurs, création des contrats, } \\
\text { validation des paiements des fournisseurs, gestion } \\
\text { capacitaire des fournisseurs, négociation des } \\
\text { conditions d'achat... }\end{array}$ \\
\hline & & Approvisionneur & $\begin{array}{l}\text { Mise à disposition des pièces conformes, } \\
\text { optimisation des niveaux des stocks et évaluation } \\
\text { de la qualité logistique des fournisseurs. }\end{array}$ \\
\hline \multirow[t]{2}{*}{ B } & \multirow[t]{2}{*}{$\begin{array}{l}\text { Fabrication } \\
\text { de faisceaux } \\
\text { électriques }\end{array}$} & $\begin{array}{l}\text { North Africa } \\
\text { Cluster Central } \\
\text { Freight } \\
\text { Coordinator }\end{array}$ & $\begin{array}{l}\text { Contrôle de la gestion des flux logistiques des } 5 \\
\text { sites au Maroc et Tunisie, la recherche de la } \\
\text { performance globale, la redynamisation des flux } \\
\text { logistiques... }\end{array}$ \\
\hline & & $\begin{array}{l}\text { Responsable } \\
\text { Approvisionnemen } \\
\mathrm{t}\end{array}$ & $\begin{array}{l}\text { Pilotage et supervision des opérations } \\
\text { d'approvisionnement et coordination et suivi des } \\
\text { livraisons. }\end{array}$ \\
\hline \multirow[t]{3}{*}{$\mathrm{C}$} & \multirow[t]{3}{*}{$\begin{array}{l}\text { Fabrication } \\
\text { de faisceaux } \\
\text { électriques }\end{array}$} & Manager logistique & 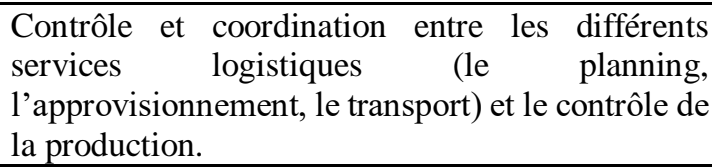 \\
\hline & & Planificateur & $\begin{array}{l}\text { Réception et traitement des commandes, } \\
\text { établissement du MRP et du plan de production, } \\
\text { lancement des ordres de fabrication et émission du } \\
\text { plan d'embarquement au service d'expédition. }\end{array}$ \\
\hline & & Approvisionneur & $\begin{array}{l}\text { Gestion des commandes des composants, gestion } \\
\text { des changements techniques, traitement des } \\
\text { obsolètes, gestion des réclamations. }\end{array}$ \\
\hline \multirow[t]{3}{*}{$\bar{D}$} & \multirow[t]{3}{*}{$\begin{array}{l}\text { Fabrication } \\
\text { des faisceaux } \\
\text { électriques }\end{array}$} & $\begin{array}{l}\text { Responsable de } \\
\text { planification de la } \\
\text { production et JIT }\end{array}$ & $\begin{array}{l}\text { Assurer avec une équipe des planificateurs de } 11 \\
\text { personnes la planification générale de l'entreprise } \\
\text { (la planification de la matière, la mise en œuvre du } \\
\text { plan de fabrication, et l'élaboration des plans } \\
\text { d'action s'il y a une déviation...) }\end{array}$ \\
\hline & & Approvisionneur & $\begin{array}{l}\text { Traitement des MRP, gestion des priorités des } \\
\text { composants, gestion des increases et decreases, } \\
\text { gestion du stock et inventaire. }\end{array}$ \\
\hline & & IT Manager & $\begin{array}{l}\text { La planification, le pilotage, le développement, } \\
\text { l'implémentation et l'entretien des systèmes } \\
\text { informatiques et de télécommunications de } \\
\text { l'entreprise. }\end{array}$ \\
\hline \multirow[t]{3}{*}{$\mathrm{E}$} & \multirow{3}{*}{$\begin{array}{l}\text { Fabrication } \\
\text { des faisceaux } \\
\text { électriques }\end{array}$} & $\begin{array}{l}\text { Responsable } \\
\text { Transport et douane }\end{array}$ & $\begin{array}{l}\text { Gestion des flux de transport actuels et éventuels, } \\
\text { gestion et suivi du volet transit et douane... }\end{array}$ \\
\hline & & Approvisionneur & $\begin{array}{l}\text { Passation des commandes et leurs suivis avec le } \\
\text { panel des fournisseurs, gestion des stocks... }\end{array}$ \\
\hline & & Key User & $\begin{array}{l}\text { Analyser et traiter les incidents du SI utilisé, ainsi } \\
\text { que ceux de l'infrastructure réseau et des matériels } \\
\text { IT et animer les formations utilisateurs. }\end{array}$ \\
\hline $\mathrm{F}$ & $\begin{array}{l}\text { Production } \\
\text { des systèmes }\end{array}$ & Responsable Achat & $\begin{array}{l}\text { Gestion des Appels d'offre, suivi de la } \\
\text { performance fournisseurs, la mise en place et le }\end{array}$ \\
\hline
\end{tabular}




\begin{tabular}{|c|c|c|c|}
\hline & \multirow{3}{*}{$\begin{array}{l}\text { de sécurité et } \\
\text { structures } \\
\text { associées (air } \\
\text { bags, ceintur } \\
\text { es de sécurité, } \\
\text { volants ...) }\end{array}$} & & $\begin{array}{l}\text { suivi d'indicateurs de performance, Optimisation } \\
\text { de l'utilisation du SI pour suivre les commandes et } \\
\text { sécuriser les approvisionnements des sites. }\end{array}$ \\
\hline & & $\begin{array}{l}\text { Responsable } \\
\text { commercial }\end{array}$ & $\begin{array}{l}\text { Garantir et développer les performances } \\
\text { commerciales, s'assurer si les clients ont reçu leurs } \\
\text { commandes convenablement et participer à la } \\
\text { reverse logistique. }\end{array}$ \\
\hline & & Manager logistique & $\begin{array}{l}\text { Optimiser la mise en place et le lancement des } \\
\text { programmes de fabrication tout en assurant une } \\
\text { gestion optimale du stock et une expédition à } \\
\text { temps aux clients. }\end{array}$ \\
\hline $\mathrm{G}$ & $\begin{array}{l}\text { Prestataire de } \\
\text { service } \\
\text { logistique } \\
\text { pour le } \\
\text { secteur } \\
\text { automobile }\end{array}$ & $\begin{array}{l}\text { Operations } \\
\text { Manager }\end{array}$ & $\begin{array}{l}\text { Gestion opérationnelle et supervision des } \\
\text { opérations et du bon fonctionnement de } \\
\text { l'exploitation, gestion et suivi de l'ensemble de } \\
\text { l'activité Route transitant par le port de Tanger } \\
\text { MED quel que soit la destination/provenance des } \\
\text { véhicules... }\end{array}$ \\
\hline Total & \multicolumn{3}{|c|}{7 entreprises et 17 entretiens } \\
\hline
\end{tabular}

\section{Collecte des données}

Pour mener à bien la phase de la collecte des données, nous nous sommes basés sur la méthodologie proposée par Ganassali (2014) concernant la collecte de données par entretiens. Ces entretiens ont été ensuite enregistrés et retranscrits intégralement afin de pouvoir les analyser dans un logiciel d'analyse textuelle.

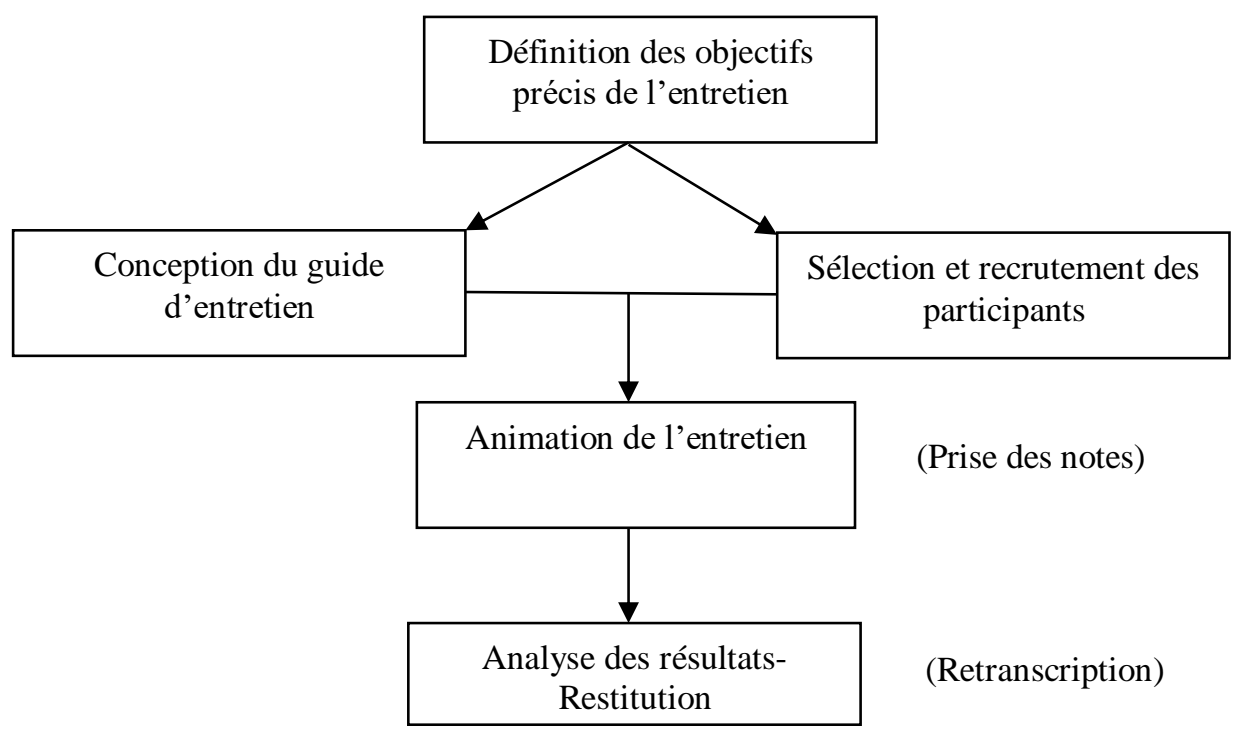

Figure 2: Schéma général de la mise en œuvre des méthodes par entretien

(S. Ganassali, 2014, P.3) 
Notre choix de méthode de collecte de données s'est porté principalement sur l'entretien semi-directif en face à face, en utilisant un guide d'entretien (voir annexe 1) suivant une série des thèmes. Les interviewés sont les différents logisticiens et décideurs issus des sept entreprises de l'industrie automobile auprès desquels nous avons pu relever les aspects les plus récurrents et les plus intéressants, qui influencent le niveau de la perception des utilisateurs SI, ainsi que leur adaptation au contexte particulier que constitue la résilience de la supply chain dans l'industrie automobile.

Notre guide d'entretien est organisé autour de quatre thèmes principaux :

- Les différentes incertitudes liées à la supply chain automobile,

- Les capacités du système d'information,

- Les antécédents de la résilience,

- La résilience de la chaine logistique.

La dernière étape des méthodes par entretiens est dédiée à l'analyse des résultats obtenus après le recueil des données. La méthode utilisée pour analyser les entretiens individuels semi-directifs est l'analyse de contenu à l'aide du logiciel d'analyse qualitative NVIVO 10.

Ce logiciel permet de gérer, de mettre en forme et de donner un sens aux données qualitatives (krief \& Zardet, 2013). Notre choix de cet outil est justifié par sa souplesse d'utilisation et sa capacité à faciliter le croisement des données après codage. Les principales étapes de l'Analyse de Contenu sont les suivantes :

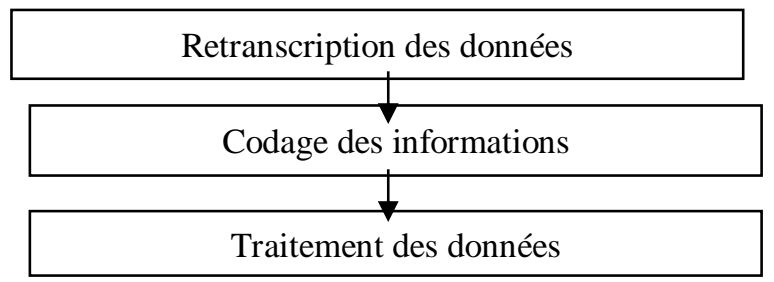

Figure 3 : Etapes de l'Analyse des données qualitatives (Andreani \& Conchon, 2005, P.17)

Conformément à notre proposition d'avoir une vision élargie des liens entre l'usage des systèmes d'information et la résilience de la chaine logistique, nous avons codé et catégorisé ces liens à partir de l'analyse de la perception des utilisateurs quant à leur usage des systèmes d'information.

L'analyse de nos études de cas est basée sur les données qualitatives recueillies lors des entretiens. Les données sont par la suite traitées à l'aide du logiciel d'analyse textuelle NVIVO. Nous avons ainsi créé dans le logiciel des catégories issues de la littérature, mais aussi issues des données empiriques. 


\section{Resultats Et Discussions}

Cette partie est dédiée à la présentation des différents résultats obtenus du traitement des données qualitatives, et ce à travers une description des catégories de codage des liens, entre l'usage du système d'information et la résilience de la chaine logistique, issues des verbatims les plus représentatifs dans les propos des différents répondants des sept entreprises étudiées (Les exemples des principaux verbatims associés aux différentes catégories sont présentés dans l'annexe 2).

\section{La perception des incertitudes liées à la chaîne logistique}

Le premier objectif de cette étude est d'explorer les différentes incertitudes liées aux supply chains automobiles, en reprenant la littérature afin de former le prisme d'analyse des résultats empiriques; il s'agit des incertitudes environnementales, des incertitudes partenariales et des incertitudes des tâches.

\section{Les incertitudes environnementales}

La revue de littérature sur les incertitudes environnementales des chaînes logistiques nous permet de relever plusieurs types d'incertitudes environnementales (incertitudes de la demande, de l'approvisionnement, des systèmes d'information, de la production...) (Duncan, 1972 ; Christopher et Towill, 2000; Feitzinger et Lee, 1997, Chopra et Meindl, 2010, Lysonski et al., 1995).

La visualisation des perceptions des acteurs interrogés concernant les incertitudes environnementales, liées à leur chaîne logistique couplée à une revue de la littérature en la matière, nous a montré qu'il est difficile d'utiliser une typologie d'incertitudes déjà existante. C'est ainsi que nous avons développé une typologie qui s'adaptera davantage à notre étude empirique.

Dans cette optique, et sur la base des propos des répondants, nous choisissons de considérer les incertitudes environnementales suivantes :

- Incertitudes liées à l'aval (demande et distribution)

- Incertitudes liées à l'amont de la chaine logistique (approvisionnement)

- Incertitudes internes (production et usinage)

- Incertitudes liées aux systèmes d'information

L'enquête empirique concernant les incertitudes environnementales liées à l'amont, à l'interne et à l'aval de la chaine, a fait révéler des catégories d'incertitudes déjà discutées dans la littérature et presque similaires dans la plupart des entreprises interviewées. Cependant, pour la catégorie des incertitudes liées au SI ; en plus des catégories déjà discutées dans la littérature, l'analyse des réponses des interviewés a fait émerger deux nouvelles catégories 
d'incertitudes liées au SI ; il s'agit de la multiplicité des applications et des fonctionnalités non maîtrisées.

Tableau 2 : Synthèse de l'analyse des données liées aux incertitudes environnementales

\begin{tabular}{|c|c|c|}
\hline Thème & Catégories & Sous catégories \\
\hline \multirow{16}{*}{$\begin{array}{l}\text { Incertitudes } \\
\text { environnementales }\end{array}$} & \multirow{2}{*}{$\begin{array}{l}\text { Incertitudes } \\
\text { liées à l'aval }\end{array}$} & $\begin{array}{l}\text { Volatilité et imprévisibilité de la demande des } \\
\text { clients }\end{array}$ \\
\hline & & Impondérables liés à la distribution \\
\hline & \multirow{3}{*}{$\begin{array}{l}\text { Incertitudes } \\
\text { internes }\end{array}$} & Non fiabilité de la planification de la production \\
\hline & & Complexité de la gestion du stock \\
\hline & & Impondérables liés à l'usine \\
\hline & \multirow{4}{*}{$\begin{array}{l}\text { Incertitudes } \\
\text { liées à } \\
\text { l'amont }\end{array}$} & Non fiabilité des fournisseurs \\
\hline & & Approvisionnement global \\
\hline & & Mono-Souricng \\
\hline & & Impondérables liés au transport \\
\hline & \multirow{7}{*}{$\begin{array}{l}\text { Incertitudes } \\
\text { liées au SI }\end{array}$} & Mauvaise qualité de l'information \\
\hline & & Absence de synchronisation des données \\
\hline & & Manque de fiabilité technique \\
\hline & & Réactivité insuffisante du support informatique \\
\hline & & Manque de fiabilité technique \\
\hline & & Multiplicité des applications \\
\hline & & Fonctionnalités non maîtrisées \\
\hline
\end{tabular}

\section{Les incertitudes partenariales}

S'agissant des incertitudes partenariales, l'analyse des résultats a montré que l'établissement des relations inter-organisationnelles des entreprises de l'industrie automobile étudiées est fondé sur deux principales dimensions, considérées comme antécédents importants pour la réussite des relations inter-organisationnelles. Il s'agit de la confiance et de l'engagement réciproque. Ces deux dimensions sont parmi les principaux facteurs largement discutés dans la littérature, pouvant permettre à l'entreprise de réduire ses incertitudes envers ses partenaires (Achrol et al., 1990; N. Venkatraman, 1995; Suhong et Binshan, 2006 ).

\section{Les incertitudes des tâches}

L'analyse des résultats empiriques a fait révéler deux principales catégories d'incertitudes liées à la tâche : il s'agit de la variété et de l'interdépendance des tâches. Dans toutes les entreprises étudiées, les acteurs mentionnent que la chaine logistique automobile est un ensemble des maillons interreliés en interne et en externe; la variété et l'interdépendance des tâches sont les principales variables caractérisant la supply chain automobile; ces deux variables communément discutées dans la littérature augmentent les incertitudes dans l'exécution du travail (Galbraith, 1973; Tushman et Nadler, 1978; Daft et Lengel, 1986). 


\section{Les capacités du système d'information}

Le deuxième objectif de cette étude est d'étudier les capacités du SI à répondre aux différentes incertitudes des supply chains automobiles et à contribuer à leurs capacités résilientes.

Le tableau ci-dessous présente les différents outils technologiques utilisés par les sept entreprises, qui leur permettent de communiquer de façon automatique avec d'autres entreprises en amont ou en aval de la chaîne logistique.

Tableau 3: Description des outils technologiques utilisés

\begin{tabular}{|c|c|c|}
\hline Entreprise & SI & Description \\
\hline \multirow{7}{*}{$\mathbf{A}$} & SAP & $\begin{array}{l}\text { ERP avec le module SCOPP (Supplier Coopération for Purchasing } \\
\text { Performance) dédié à la gestion des achats. }\end{array}$ \\
\hline & EDI & Moyen d'échange des données entre l'entreprise A et ses fournisseurs. \\
\hline & Portail B2B & $\begin{array}{l}\text { Outil accessible à l'ensemble des fournisseurs qui partagent une } \\
\text { interface avec l'entreprise quel que soit leur implantation géographique. }\end{array}$ \\
\hline & Intranet & $\begin{array}{l}\text { Application partagée en interne qui facilite à chaque département } \\
\text { l'accès aux différentes informations. }\end{array}$ \\
\hline & ANPQP & Application dédiée au démarrage des nouveaux projets fournisseurs. \\
\hline & $\mathrm{ADCP}$ & $\begin{array}{l}\text { Application dédiée à la gestion des capacités des fournisseurs en termes } \\
\text { du volume. }\end{array}$ \\
\hline & EPDC & Application dédiée à la gestion des packagings. \\
\hline \multirow{6}{*}{ B } & SAP & Gestion intégrée de l'activité de l'entreprise B. \\
\hline & EDI & Echange des données entre l'entreprise B et ses partenaires. \\
\hline & GMMT & $\begin{array}{l}\text { Application spécialisée pour le suivi des indicateurs de performance de } \\
\text { l'approvisionnement, des niveaux des stocks. }\end{array}$ \\
\hline & $\begin{array}{l}\text { Transport } \\
\text { Order } \\
\text { Notification }\end{array}$ & $\begin{array}{l}\text { Application partagée avec les prestataires logistiques (transporteurs) } \\
\text { pour réserver planifier, organiser et suivre les opérations du transport. }\end{array}$ \\
\hline & TMS & $\begin{array}{l}\text { Outil d'aide à la gestion du transport, il répond principalement aux } \\
\text { besoins de traçabilité des livraisons et d'optimisation du transport. }\end{array}$ \\
\hline & $\begin{array}{l}\text { Feed Back } \\
\text { System }\end{array}$ & $\begin{array}{l}\text { Application pour communiquer entre l'approvisionnement et le magasin } \\
\text { de réception. }\end{array}$ \\
\hline \multirow{4}{*}{$\mathbf{C}$} & XPPS & ERP qui permet de supporter l'activité logistique de l'entreprise C. \\
\hline & Intranet & Portail qui englobe l'ensemble des procédures de l'entreprise. \\
\hline & CRM & $\begin{array}{l}\text { Outil utilisé par le customer service pour tous ce qui est en relation avec } \\
\text { les besoins des clients pour les transférer au service planning. }\end{array}$ \\
\hline & EDI & $\begin{array}{l}\text { Transfert des documents commerciaux avec les partenaires de } \\
\text { l'entreprise C. }\end{array}$ \\
\hline \multirow{4}{*}{ D } & AS400 & ERP dédié à supporter l'activité logistique de l'entreprise D. \\
\hline & PLS & $\begin{array}{l}\text { Système dédié à la gestion des produits finis avec une interface avec } \\
\text { AS400. }\end{array}$ \\
\hline & MAO & $\begin{array}{l}\text { Application dédiée à la préparation des commandes pour optimiser le } \\
\text { temps et les capacités en ressources et équipement. }\end{array}$ \\
\hline & EDI & $\begin{array}{l}\text { La réception des commandes des clients et la passation des commandes } \\
\text { aux fournisseurs. }\end{array}$ \\
\hline
\end{tabular}




\begin{tabular}{|l|l|l|}
\hline \multirow{4}{*}{ E } & QAD & $\begin{array}{l}\text { ERP utilisé au sein de l'entreprise E, il intègre tous les maillons de la } \\
\text { chaine de création de valeur (Production, Logistique, Quality, ...). }\end{array}$ \\
\cline { 2 - 3 } & Transvoyant & $\begin{array}{l}\text { Application dédiée à gérer le tracking des camions depuis les usines de } \\
\text { l'entreprise E vers le client final. }\end{array}$ \\
\cline { 2 - 3 } & EDI & Echange des données entre l'entreprise E et ses partenaires. \\
\hline \multirow{3}{*}{ F } & XPPS & ERP dédié à supporter l'activité logistique de l'entreprise F. \\
\cline { 2 - 4 } & EDI & Echange des données entre l'entreprise F et ses partenaires. \\
\hline \multirow{4}{*}{ G } & AS400 & $\begin{array}{l}\text { Système de gestion intégral dédié à supporter l'ensemble des activités } \\
\text { de l'entreprise. }\end{array}$ \\
\cline { 2 - 4 } & EDI & Echange des données entre l'entreprise G et ses partenaires. \\
\cline { 2 - 3 } & «Trust \& & $\begin{array}{l}\text { Système partagé en ligne avec les clients leur permettant de faire des } \\
\text { booking, et le suivi de la traçabilité de leurs envois. }\end{array}$ \\
\hline
\end{tabular}

L'analyse des résultats a fait révéler des capacités du SI déjà citées dans la littérature (Tableau 4), il s'agit de la capacité du traitement de l'information, la capacité de la communication et du partage de l'information en interne et en externe de l'entreprise, et la capacité de prise des décisions efficaces (Galbraith, 1977; Tushman et Nadler, 1978 ; Moberg et al., 2002 ; Pan et al., 2012).

Tableau 4 : Synthèse de l'analyse des données liées aux capacités du SI

\begin{tabular}{|l|l|}
\hline Le thème & Les catégories \\
\hline \multirow{4}{*}{ Capacités du SI } & Capacité du traitement de l'information \\
\cline { 2 - 2 } & Communication et partage d'informations en interne \\
\cline { 2 - 2 } & Communication et partage d'informations en externe \\
\cline { 2 - 2 } & Capacité d'aide à la décision \\
\hline
\end{tabular}

Selon les interviewés, le système d'information est capable de traiter les informations en collaboration, de partager les informations en temps réel entre les différents acteurs internes et externes de la chaine logistique, et de permettre la prise des décisions collectives.

Pour que le SI soit en mesure de remplir les quatre capacités évoquées, les interviewés ont mis l'accent sur l'importance de la qualité de la formation des utilisateurs en SI et l'engagement de la direction à offrir un meilleur service aux utilisateurs du SI, et ce dans le but de fournir aux utilisateurs les compétences nécessaires pour faire face aux incertitudes de leurs supply chains.

\section{Les antécédents de la résilience}

Le troisième objectif de cette étude fait référence à l'identification des principaux antécédents de la résilience de la chaine logistique automobile. Les résultats de l'étude laissent constater deux principaux antécédents largement débattus dans la littérature: Il s'agit de l'intégration et de la flexibilité (Christopher, 2005 ; Flynn et al., 2010 ; Prater et al., 2001). L'ensemble des entreprises étudiées ont confirmé que le recours à des pratiques relevant de 
l'intégration et de la flexibilité permet de développer la résilience de la chaîne logistique automobile (voir tableau 5).

Tableau 5 : Synthèse de l'analyse des données liées aux antécédents de la résilience

\begin{tabular}{|l|l|}
\hline Le thème & Les catégories \\
\hline \multirow{4}{*}{ Intégration } & Intégration interne \\
\cline { 2 - 2 } & Intégration des clients \\
\cline { 2 - 2 } & Intégration des fournisseurs \\
\hline \multirow{4}{*}{ Flexibilité } & Flexibilité des contrats \\
\cline { 2 - 2 } & Utilisation des composants standards \\
\cline { 2 - 2 } & Flexibilité des collaborateurs \\
\cline { 2 - 2 } & Flexibilité dans le changement de modes du transport \\
\cline { 2 - 2 } & Approvisionnement à sources multiples \\
\hline
\end{tabular}

L'intégration via ses mécanismes internes et externes est perçue par l'ensemble des entreprises de l'industrie automobile comme une stratégie à la fois proactive et réactive, permettant de renforcer les capacités résilientes de la supply chain automobile (Galbraith 1973; Daft et Lengel, 1986).

La flexibilité quant à elle, a été adoptée par les entreprises de l'industrie automobile comme une stratégie visant, avec les cinq catégories des pratiques flexibles déjà citées dans la littérature, à faire face aux différentes incertitudes; dont l'approvisionnement à sources multiples constitue une pratique fondamentale contribuant au développement de la flexibilité de la chaine logistique (Tang et Tomlin, 2008 ; Sawhney, 2006 ; Tachizawa et Gimenez, 2010 ; Timothy J. Petit, M.S, 2008). En effet, nonobstant l'apport de la pratique de l'approvisionnement à sources multiples dans le renforcement de la flexibilité de la chaine logistique, cette dernière n'a pas été abordée par l'ensemble des entreprises interviewées, et ce malgré les répercussions néfastes que peuvent avoir les sources uniques sur la résilience de leurs chaines logistiques.

\section{La résilience de la chaine logistique}

Le quatrième objectif de cette étude exploratoire est de mettre en lumière la façon dont les entreprises de l'industrie automobile perçoivent la résilience de leurs supply chains, tout en étudiant l'apport du SI dans la contribution aux capacités résilientes de leurs supply chains.

Ainsi, malgré l'ambigüité que les interviewés avaient au début des entretiens sur la notion de la résilience de la chaine logistique, ces derniers ont arrivé à l'associer aux quatre catégories des capacités résilientes déjà discutées dans la littérature, à savoir : la capacité d'anticipation, la capacité de réponse, la capacité de rétablissement et la capacité d'apprentissage (Rice \& Caniato ,2003 ; Sheffi \& Rice, 2005; Blackhurst et al., 2005; Tang, 2006 ; Ponomarov et Holcomb, 2009 ; Ritchie et Brindley, 2007) tout en mettant en évidence le rôle joué par le SI dans les quatre capacités résilientes. 
Tableau 6: Synthèse de l'analyse des données liées à la résilience de SC

\begin{tabular}{|l|l|}
\hline Le thème & Les catégories \\
\hline \multirow{4}{*}{ Résilience de la supply chain } & Capacité d'anticipation \\
\cline { 2 - 2 } & Capacité de réponse \\
\cline { 2 - 2 } & Capacité de rétablissement \\
\cline { 2 - 2 } & Capacité d'apprentissage \\
\hline
\end{tabular}

Selon l'ensemble des interviewés, la capacité d'anticipation correspond aux efforts mobilisés par les acteurs internes et externes de la chaine logistique avant que la perturbation ne survienne, il s'agit essentiellement de l'importance de la collaboration intra et inter organisationnelle au sein de la supply chain et du rôle clé du système d'information en matière de partage d'information entre les différents acteurs.

Quant à la capacité de réponse, l'ensemble des interviewés ont mis l'accent sur la capacité de l'entreprise à réagir face à l'événement perturbateur, et ce grâce à l'intégration et à la flexibilité des différents acteurs permettant de faciliter la prise de décision face aux perturbations.

La capacité de rétablissement correspond à son tour au processus de retour à l'état d'origine de la chaîne logistique après avoir été perturbée, voire à un état mieux que l'original. Les réponses trouvées aux perturbations rencontrées amènent souvent les entreprises interrogées à revenir à l'état normal de fonctionnement.

Dans la même lignée des capacités résilientes évoquées, les interviews ont souligné l'importance de l'apprentissage dans le développement de la résilience et du rôle du SI dans le transfert des connaissances, en interne et externe des entreprises, pour anticiper et répondre à une éventuelle future perturbation.

Après avoir discuté et analysé l'apport des différentes capacités de gestion résiliente des perturbations dans la chaine logistique, les différents interviewés ont proposé une définition de la résilience selon leur perception et leur contexte d'intervention.

Les définitions de la résilience de la supply chain évoquées par les interviewés permettent d'analyser et de contextualiser la résilience de la supply chain dans le secteur de l'industrie automobile. Pour l'ensemble des interviewés, il est important d'aborder la résilience en combinaison avec les incertitudes, les perturbations et les capacités. Ainsi, la résilience de la supply chain est considérée comme une série de réponses adaptatives dans une approche en plusieurs étapes, intégrant des capacités avant, pendant et après les perturbations ; il s'agit de la capacité de l'anticipation des perturbations, la capacité de réponse aux perturbations, la capacité de rétablissement après les perturbations et la capacité d'apprentissage pour anticiper toute éventuelle répétition des perturbations. Enfin, en se basant sur les définitions de la résilience proposées par les différents interviewés, nous avons formulé une 
définition synthétique de la résilience de la chaine logistique adaptée au contexte de l'industrie automobile comme suit : «La résilience est la capacité de la chaîne logistique à anticiper les perturbations, à répondre à ces perturbations et à se rétablir après avoir été perturbée. L'objectif est de permettre à la chaine logistique de revenir à son état d'origine ou à un état plus souhaitable après avoir subi une perturbation, tout en capitalisant les connaissances acquises à l'issue des expériences pour anticiper leur répétition dans le futur».

\section{Proposition d'un modèle conceptuel de recherche}

En résumé, notre recherche qualitative et exploratoire nous a permis de contextualiser notre modèle sur le terrain au niveau des entreprises de l'industrie automobile au Maroc.

La figure suivante illustre l'adaptation du modèle de Elkharraz et Moukadem (2019) au contexte marocain, sur la base d'une étude qualitative exploratoire.

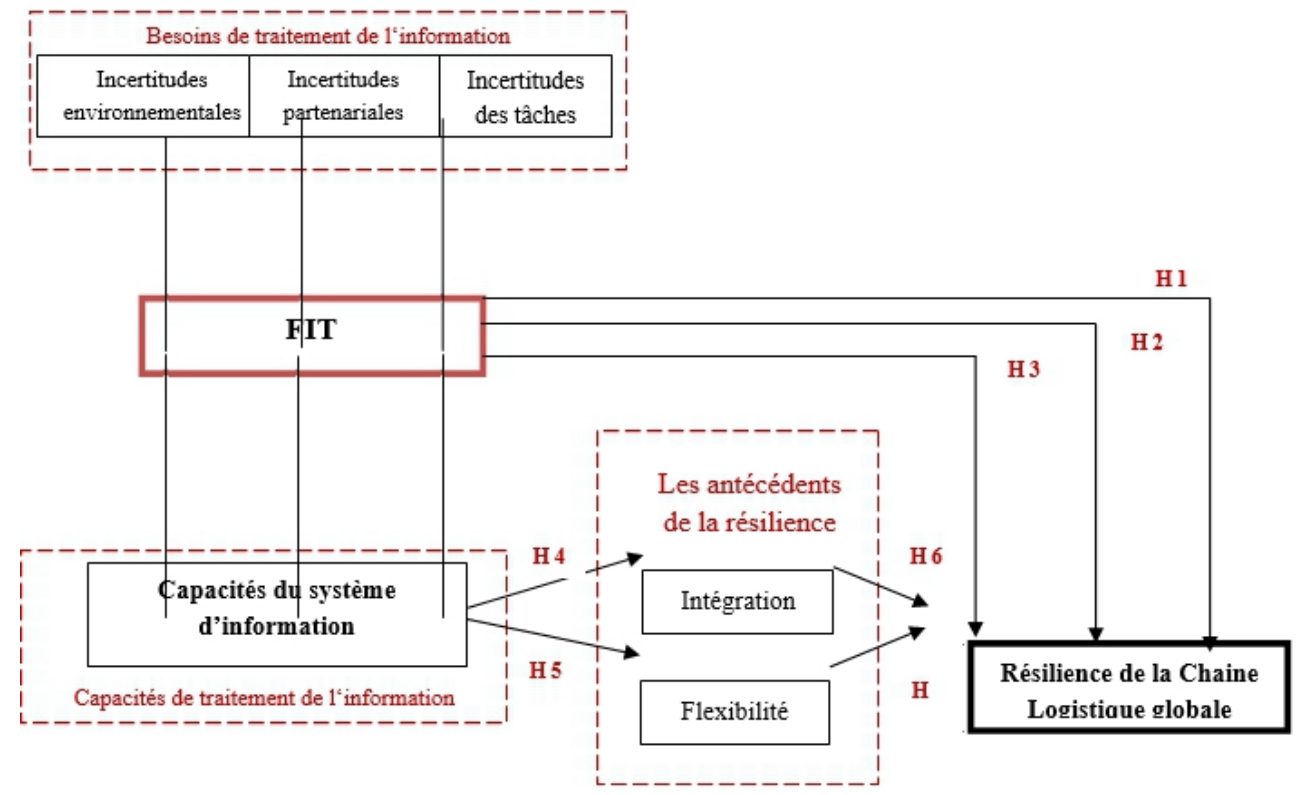

Figure 4: Modèle conceptuel de l'étude de la contribution de l'usage des SI à la résilience de la chaine logistique globale

\section{Conclusion}

L'objectif de cet article était de présenter et de justifier le cadre empirique mobilisé, ainsi que de proposer un modèle conceptuel de recherche issu d'une approche qualitative exploratoire menée auprès de sept entreprises opérant dans le secteur automobile au Maroc.

Après une revue de littérature qui a permis de concevoir un modèle théorique de recherche, susceptible d'expliquer l'utilité de l'usage des 
systèmes d'information dans la résilience de la chaine logistique globale, une enquête qualitative a été menée auprès des sept entreprises opérant dans le secteur automobile au Maroc. La phase qualitative et exploratoire a été d'une grande contribution pour la recherche, cette dernière a été intégrée au regard de la nécessité de se rapprocher du terrain de l'étude, afin de relever auprès des praticiens et des spécialistes du métier, les aspects les plus récurrents et les plus intéressants qui influencent le niveau de la perception des utilisateurs des SI, ainsi que leur adaptation au contexte particulier que constitue la résilience de la supply chain dans l'industrie automobile.

Les données collectées à l'aide du guide d'entretien ont permis de développer une nouvelle typologie d'incertitudes adaptée au contexte des supply chains automobiles. Ensuite, l'analyse des résultats empiriques a fait émerger des capacités des systèmes d'information permettant de faire face aux incertitudes des supply chains et d'accroître leurs résiliences. Enfin, l'étude exploratoire a mis en lumière la façon dont les entreprises de l'industrie automobile perçoivent la résilience de leurs supply chains, ce qui a permis de proposer une définition synthétique de la résilience de la chaine logistique adaptée au contexte de l'industrie automobile.

Nous proposons à travers notre travail d'apporter une contribution aussi bien au niveau théorique que managérial. Au niveau théorique, nous avons proposé un modèle conceptuel permettant l'évaluation de la contribution des SI à la résilience de la chaine logistique globale. Au niveau managérial, nous avons adapté notre modèle théorique aux entreprises de l'industrie automobile, cette adaptation permettra aux managers logistiques de celles-ci, d'entreprendre des actions managériales afin d'accroître la capacité résiliente de leurs supply chains.

La limite principale de cette recherche réside dans la généralisation des résultats à partir d'une population qui peut paraitre restreinte. A cet effet, il serait judicieux de compléter notre étude exploratoire par une étude quantitative confirmatoire, sur un échantillon plus vaste d'entreprises, afin d'opérationnaliser les construits de la recherche, et de valider les instruments de mesure des différentes variables du modèle conceptuel de la recherche

\section{References:}

1. Achrol, R. S. (1991). «Evolution of the marketing organization: new forms for turbulent environments ». Journal of Marketing, 55(4), 7793.

2. Al Meriouh, Y., Elkharraz, A., \& Al Barrak, F. (2017). «Etude de l'impact des systèmes d'information sur la gestion des approvisionnements dans l'industrie automobile ». Revue Marocaine de Management, Logistique et Transport, (2). 25-45. 
3. ANDREANI J.C, CONCHON F. (2005), « Fiabilité et Validité des Enquêtes Qualitatives. Un état de l'art en Marketing », Revue Française du Marketing, $\mathrm{N}^{\circ}$ 201, 2005/1-5, p. 5-21.

4. Aubert B.A. \& Dussart A., (2002). « Systèmes d'information interentreprises ». Rapport Bourgogne du CIRANO, 2002RB-01.

5. Bensaou, M., \& Venkatraman, N. (1996). « Inter-organizational relationships and information technology: A conceptual synthesis and a research framework ». European Journal of Information Systems, 5(2), 84-91.

6. Blackhurst*, J., Craighead, C. W., Elkins, D., \& Handfield, R. B. (2005). « An empirically derived agenda of critical research issues for managing supply-chain disruptions ». International Journal of Production Research, 43(19), 4067-4081.

7. Blackhurst, J., Dunn, K. S., \& Craighead, C. W. (2011). « An empirically derived framework of global supply resiliency ». Journal of Business Logistics, 32(4), 374-391.

8. Boissinot, A. (2010). « L'influence d'asymétrie d'information entre chargeur et prestataires de services logistiques dans l'industrie automobile ». Logistique \& Management, 18(2), 85-96.

9. Bostrom, P. R et Heinen, J. S., (1977). « MIS Problems and Failures: A Socio-Technical Perspective Part I: The Causes ». MIS Quarterl, p.17-32.

10. Boubker, O., \& Chafik, K. (2015). « Elaboration d'un modèle théorique d'évaluation de la contribution de systèmes d'information logistiques à la performance et la maturité des processus logistiques ». Xème Conférence Internationale : Conception et Production Intégrées. Presented at the Tanger, Morocco. Retrieved from https://hal.archivesouvertes.fr/hal-01260767

11. Boubker, O., \& Chafik, K. (2016). « Comprendre le rôle des systèmes d'information dans l'amélioration de la performance et la maturité des processus logistiques : étude qualitative auprès de six entreprises de l'industrie automobile et aéronautique au Maroc ». European Scientific Journal, ESJ, 12(28), 497-429. doi: 10.19044/esj.2016.v12n28p397

12. Boubker, O. (2017). «Évaluation de la contribution des systèmes d'information à la performance et à la maturité des processus logistiques : Cas des entreprises de l'industrie automobile et aéronautique au Maroc ». (Thèse de doctorat en Sciences de Gestion. Université Abdelmalek Essaadi, ENCG de Tanger). 
13. Boubker, O., \& Chafik, K. (2017). «An investigation of the relationship between logistics information quality and LIS use based on structural equation modelling approach: A case study of automotive and aerospace companies in Morocco». New Trends and Issues Proceedings on Humanities and Social Science., 4(10), 97-109.DOI: 10.18844/prosoc.v4i10.3068

14. Brusset, X., \& Teller, C. (2017). «Supply chain capabilities, risks, and resilience ». International Journal of Production Economics, 184, 5968. https://doi.org/10.1016/j.ijpe.2016.09.008

15. Carvalho, H., Maleki, M., \& Cruz-Machado, V. (2012). « The links between supply chain disturbances and resilience strategie »s. International Journal of Agile Systems and Management, 5(3), 203234.

16. Charmaz, K. (2006). «Constructing grounded theory: A practical guide through qualitative analysis ». Sage Publications.

17. Chen, G. (2013). « On the competitiveness of aviation logistics industry ». 2013 Fourth International Conference on Education and Sports Education (ESE 2013), Hong Kong, 11-12.

18. Chopra, S., \& Meindl, P. (2006). « Supply chain performance: Achieving strategic fit and scope ». Supply Chain Management: Strategy, Planning, and Operations, 22-42.

19. Chopra, Sunil, \& Sodhi, M. S. (2004). « Supply-chain breakdown ». MIT Sloan Management Review, 46(1), 53-61.

20. Chowdhury, M. M. H., \& Quaddus, M. (2016). « Supply chain readiness, response and recovery for resilience ». Supply Chain Management: An International Journal, 21(6), 709-731.

21. Christopher, M., \& Peck, H. (2004). « Building the resilient supply chain ». The International Journal of Logistics Management, 15(2), 114.

22. Christopher M., (2005). «Logistics and Supply Chain Management ». Fourth Edition, FT Prentice Hall.

23. Christopher, M., \& Towill, D. R. (2000). « Supply chain migration from lean and functional to agile and customised ». Supply Chain Management: An International Journal, 5(4), 206-213.

24. COMTD (Committee on Trade and Development, (1996) « Participation of developing countries in World Trade: Overview of major trends and underlying factors », World Trade Organization. 
25. Ouabouch, L. \& Paché, G.,(2014). «Risk Management in the Supply Chain: Characterization and Empirical Analysis ».The Journal of Applied Business Research, p.329-340.

26. Daft, R. L., \& Lengel, R. H. (1986). « Organizational information requirements, media richness and structural design ». Management Science, 32(5), 554-571.

27. De Corbière, F., Rowe, F., \& Wolff, F.-C. (2012). « De l'intégration interne $\mathrm{du}$ système d'information à l'intégration du système d'information de la chaîne logistique ». Systemes d'information Management, 17(1), 81-111.

28. Duncan, R. B. (1972). « Characteristics of organizational environments and perceived environmental uncertainty ». Administrative Science Quarterly, 313-327.

29. Eisenhardt, K. M. (1989). « Building theories from case study research ». Academy of Management Review, 14(4), 532-550.

30. Elkharraz, A., \& Moukadem, K. (2019). « Contribution de l'usage des systèmes d'information à la résilience de la chaine logistique globale: Élaboration d'un modèle théorique ». International Journal of Innovation and Applied Studies, 25(2), 718.

31. Feitzinger, E., \& Lee, H. L. (1997). « Mass customization at HewlettPackard: the power of postponement ». Harvard Business Review, 75, 116-123.

32. Fleury, A., \& Fleury, M. T. (2001). « Alternatives for industrial upgrading in global value chains the case of the plastics industry in Brazil ». IDS Bulletin, 32(3), 116-126.

33. Flynn, B. B., Huo, B., \& Zhao, X. (2010). « The impact of supply chain integration on performance: A contingency and configuration approach ». Journal of Operations Management, 28(1), 58-71.

34. Galbraith, J. R. (1977). «Organization design ». Addison Wesley Publishing Company.

35. Ganassali, S. (2014). «Enquêtes et analyse de données avec Sphinx: $\gg$ Livre+ plateforme interactive eText-Licence 12 mois. Pearson Education France.

36. Harrison, A., \& Van Hoek, R. I. (2008). « Logistics management and strategy: competing through the supply chain ». Pearson Education.

37. Hohenstein, N.-O., Feisel, E., Hartmann, E., \& Giunipero, L. (2015). «Research on the phenomenon of supply chain resilience: a systematic review and paths for further investigation ». International Journal of Physical Distribution \& Logistics Management, 45(1/2), 90-117. 
38. Jüttner, U. (2005). « Supply chain risk management: Understanding the business requirements from a practitioner perspective ». The International Journal of Logistics Management, 16(1), 120-141.

39. Jüttner, U., \& Maklan, S. (2011). "Supply chain resilience in the global financial crisis: an empirical study ». Supply Chain Management: An International Journal, 16(4), 246-259.

40. Klibi, W., Martel, A., \& Guitouni, A. (2010). « The design of robust value-creating supply chain networks: a critical review ». European Journal of Operational Research, 203(2), 283-293.

41. Krief, N., \& Zardet, V. (2013). «Analyse de données qualitatives et recherche-intervention ». Recherches En Sciences de Gestion, (2), 211-237.

42. Li, S., \& Lin, B. (2006). "Accessing information sharing and information quality in supply chain management ». Decision Support Systems, 42(3), 1641-1656. https://doi.org/10.1016/j.dss.2006.02.011

43. Lisein, O., \& De Zanet, F. (2013). « Stimulants organisationnels et RH de l'intrapreneuriat ». Revue Française de Gestion, (4), 141-160.

44. Lysonski, S., Levas, M., \& Lavenka, N. (1995). « Environmental uncertainty and organizational structure: a product management perspective ». Journal of Product \& Brand Management. https://doi.org/10.1108/10610429510097609

45. Markus, M. L. (2001). "Reflections on the systems integration enterprise ». Business Process Management Journal. https://doi.org/10.1108/bpmj.2001.15707caf.001

46. Mentzer, J. T., DeWitt, W., Keebler, J. S., Min, S., Nix, N. W., Smith, C. D., \& Zacharia, Z. G. (2001). « Defining Supply Chain Management ». Journal of Business Logistics, 22(2), 1-25. https://doi.org/10.1002/j.2158-1592.2001.tb00001.x

47. Moberg, C. R., Cutler, B. D., Gross, A., \& Speh, T. W. (2002). « Identifying antecedents of information exchange within supply chains ». International Journal of Physical Distribution \& Logistics Management. https://doi.org/10.1108/09600030210452431

48. Ngai, E. W. T., Chau, D. C. K., \& Chan, T. L. A. (2011). «Information technology, operational, and management competencies for supply chain agility: Findings from case studies ». The Journal of Strategic Information Systems, 20(3), 232-249. https://doi.org/10.1016/j.jsis.2010.11.002 
49. Pan, S.L., Pan, G., Leidner, D.E., (2012). « Crisis Response Information Networks ». Journal of the Association for Information Systems, 13(1), 31-56.

50. Peck, H. (2005). « Drivers of supply chain vulnerability: an integrated framework ». International Journal of Physical Distribution \& Logistics Management. https://doi.org/10.1108/09600030510599904

51. Pettit TJ, Fiksel TJJ, Croxton KL., (2010). « Ensuring Supply Chain Resilience: Development of a Conceptual Framework ». Journal of Business Logistics, 31(1), 1-2.

52. Ponomarov, S. (2012). «Antecedents and Consequences of Supply Chain Resilience: A Dynamic Capabilities Perspective ». Doctoral Dissertations.

53. Ponomarov, S. Y., \& Holcomb, M. C. (2009). «Understanding the concept of supply chain resilience ». The International Journal of Logistics Management. https://doi.org/10.1108/09574090910954873

54. Prajogo, D., \& Olhager, J. (2012). « Supply chain integration and performance: The effects of long-term relationships, information technology and sharing, and logistics integration ». International Journal of Production Economics, 135(1), 514-522. https://doi.org/10.1016/j.ijpe.2011.09.001

55. Prater, E., Biehl, M., \& Smith, M. A. (2001). «International supply chain agility - Tradeoffs between flexibility and uncertainty ». International Journal of Operations \& Production Management. https://doi.org/10.1108/01443570110390507

56. Pratt, M. G. (2009). « From the Editors: For the Lack of a Boilerplate: Tips on Writing Up (and Reviewing) Qualitative Research ». Academy of Management Journal, 52(5), 856-862. https://doi.org/10.5465/amj.2009.44632557

57. Priya Datta, P., Christopher, M., \& Allen, P. (2007). « Agent-based modelling of complex production/distribution systems to improve resilience ». International Journal of Logistics Research and Applications, 10(3), 187-203.

58. Rice, J. B., \& Caniato, F. (2003). «BUILDING A SECURE AND RESILIENT SUPPLY NETWORK ». SUPPLY CHAIN MANAGEMENT REVIEW, V. 7, NO. 5 (SEPT./OCT. 2003), P. 22 30 : ILL.

59. Ritchie, B., \& Brindley, C. (2007). « Supply chain risk management and performance ». International Journal of Operations \& Production Management. https://doi.org/10.1108/01443570710725563 
60. Samuel, K. E., \& Ruel, S. (2013). « Systèmes d'information et résilience des chaînes logistiques globales ». Systemes d'information Management, 18(1), 57-85.

61. Sawhney, R. (2006). « Interplay between uncertainty and flexibility across the value-chain: Towards a transformation model of manufacturing flexibility ». Journal of Operations Management, 24(5), 476-493. https://doi.org/10.1016/j.jom.2005.11.008

62. Tachizawa, E. M., \& Gimenez, C. (2010). « Supply flexibility strategies in Spanish firms: Results from a survey ». International Journal of Production Economics, 124(1), 214-224. https://doi.org/10.1016/j.ijpe.2009.11.020

63. Tang, C. S. (2006). « Robust strategies for mitigating supply chain disruptions ». International Journal of Logistics: Research and Applications, 9(1), 33-45.

64. Tang, C., \& Tomlin, B. (2008). « The power of flexibility for mitigating supply chain risks ». International Journal of Production Economics, 116(1), 12-27.

65. Thietart, R.-A. (2014). « Méthodes de recherche en management 4ème édition (4e édition) ». Paris: Dunod.

66. Tushman, M. L., \& Nadler, D. A. (1978). «Information Processing as an Integrating Concept in Organizational Design ». Academy of Management $\quad$ Review, 3(3), 613-624. https://doi.org/10.5465/amr.1978.4305791

67. Venkatraman, N. (1989). « The Concept of Fit in Strategy Research: Toward Verbal and Statistical Correspondence ». Academy of Management Review, 14(3), 423-444. https://doi.org/10.5465/amr.1989.4279078

68. Wang, E. T. G., Tai, J. C. F., \& Wei, H.-L. (2006). « A Virtual Integration Theory of Improved Supply-Chain Performance ». Journal of Management Information Systems, 23(2), 41-64. https://doi.org/10.2753/MIS0742-1222230203

69. Wei, H.-L., Wong, C. W. Y., \& Lai, K. (2012). « Linking interorganizational trust with logistics information integration and partner cooperation under environmental uncertainty ». International Journal of Production Economics, 139(2), 642-653. https://doi.org/10.1016/j.ijpe.2012.05.036

70. Wieland, A., \& Wallenburg, C. M. (2013). « The influence of relational competencies on supply chain resilience: a relational view ». 


\section{International Journal of Physical Distribution \& Logistics Management. https://doi.org/10.1108/IJPDLM-08-2012-0243}

71. Wu, T., Huang, S., Blackhurst, J., Zhang, X., \& Wang, S. (2013). « Supply Chain Risk Management: An Agent-Based Simulation to Study the Impact of Retail Stockouts ». IEEE Transactions on Engineering Management, 60(4), 676-686. https://doi.org/10.1109/TEM.2012.2190986

72. Yin, R. K. (1994). « Case study research: Design and methods (2nd ed.) ». Newbury Park, CA: Sage Publications.

\section{Annexes}

Annexe 1 : Guide d'entretien utilisé pour la collecte des données

\begin{tabular}{|c|c|}
\hline Thème & Exemples des questions posées lors des entretiens \\
\hline Introduction & $\begin{array}{l}\text { Identification de l'entreprise, présentation de interviewés, présentation de la } \\
\text { structure interne et externe de la supply chain... }\end{array}$ \\
\hline $\begin{array}{c}\text { Incertitudes } \\
\text { environnementales }\end{array}$ & $\begin{array}{l}\text { Quelles sont les incertitudes liées à l'aval de la supply chain? Quelles sont les } \\
\text { incertitudes liées à l'amont de la supply chain? Quelles sont les incertitudes } \\
\text { liées aux évolutions technologiques et aux systèmes d'information de la } \\
\text { supply chain? }\end{array}$ \\
\hline $\begin{array}{l}\text { Incertitudes } \\
\text { partenariales }\end{array}$ & $\begin{array}{l}\text { Comment pourriez-vous évaluer votre confiance mutuelle avec vos partenaires } \\
\text { en termes de partage d'information et de collaboration? Dans quelle mesure } \\
\text { votre entreprise s'investit-elle spécifiquement pour sa relation avec ses } \\
\text { partenaires? Pourriez-vous nous donner des exemples? Dans quelle mesure } \\
\text { vos partenaires s'investissent-ils spécifiquement pour leurs relations avec } \\
\text { votre entreprise? Pourriez-vous nous donner des exemples? }\end{array}$ \\
\hline $\begin{array}{l}\text { Incertitudes des } \\
\text { tâches }\end{array}$ & $\begin{array}{l}\text { Dans quelle mesure appliquez-vous la procédure pour exécuter vos tâches? } \\
\text { Dans quelle mesure le nombre d'exceptions et d'événements imprévus } \\
\text { complexifie-t-il l'exécution de vos tâches? } \\
\text { Dans quelle mesure les interdépendances qui relient vos tâches avec les autres } \\
\text { fonctions de l'entreprise augmentent-elles vos incertitudes lors de l'exécution } \\
\text { de votre travail? }\end{array}$ \\
\hline Capacités des SI & $\begin{array}{l}\text { Quelles sont les autres applications technologiques que vous utilisez pour } \\
\text { communiquer de façon automatique avec d'autres entreprises en amont ou en } \\
\text { aval de la chaîne logistique ? Comment pourriez-vous évaluer la capacité de } \\
\text { votre système d'information logistique à répondre à vos besoins en } \\
\text { informations requises lors de l'exécution de vos tâches? Dans quelle mesure } \\
\text { l'usage de votre système d'information logistique influe-t-il sur votre relation } \\
\text { avec vos partenaires externes en termes de communication et d'échange } \\
\text { d'informations entre les partenaires de la chaine logistique? Comment } \\
\text { pourriez-vous évaluer les programmes de formation reçus sur l'utilisation des } \\
\text { SI ? En cas des difficultés rencontrées lors de l'usage du SI, la fonction support } \\
\text { SI est-elle suffisamment disponible et réactive? }\end{array}$ \\
\hline Intégration & $\begin{array}{l}\text { Comment collaborez-vous et gérez vos relations entre les différentes fonctions } \\
\text { et sites détenues par votre entreprise ? Est-ce qu'il y a un encouragement de la } \\
\text { part de la direction en matière de collaboration interservices? Dans quelle } \\
\text { mesure votre collaboration avec vos clients permet-elle de vous donner une } \\
\text { visibilité en matière de l'anticipation de la demande des clients? }\end{array}$ \\
\hline
\end{tabular}




\begin{tabular}{|c|l|}
\hline Flexibilité & $\begin{array}{l}\text { Aviez-vous des contrats ouverts avec vos fournisseurs en matière de } \\
\text { changements des quantités, spécifications et conditions ? Vos achats en } \\
\text { matières premières/composants sont-ils utilisés dans plusieurs de vos produits } \\
\text { finis? Aviez-vous des sources multiples pour vos approvisionnements ? Dans } \\
\text { quelle mesure êtes-vous capable de vous adapter aux fluctuations de la } \\
\text { demande de vos clients? }\end{array}$ \\
\hline \multirow{2}{*}{ Résilience de la } \\
chaine logistique & $\begin{array}{l}\text { Dans quelle mesure votre entreprise avait-elle mis en place des mécanismes } \\
\text { d'anticipation et de préparation envers la perturbation? Pouvez-vous nous } \\
\text { décrire la façon dont la perturbation a été gérée? Votre chaine logistique est- } \\
\text { elle revenue à son état d'origine ou se transformer en un état plus désirable } \\
\text { après avoir été perturbée? Votre chaine logistique a pu tirer une expérience de }\end{array}$ \\
& $\begin{array}{l}\text { la perturbation? Cette expérience est-elle conservée? Comment ? Comment } \\
\text { l'usage de votre système d'information a-t-il permis à votre supply chain de } \\
\text { capitaliser cette expérience et de développer la capacité d'apprentissage? }\end{array}$ \\
\hline
\end{tabular}

\section{Annexe 2 : Exemples des principaux verbatims associés aux différentes catégories}

Verbatims liés aux incertitudes environnementales

Verbatims associés aux incertitudes liées à l'aval de la chaine logistique

«...on souffre toujours de la fluctuation de la demande, parfois même on reçoit des commandes anticipées de la part de nos clients ce qui nous perturbe tous nos programmes déjà planifiés... » (Entreprise C, Manager logistique).

«... on recoure aux expéditions urgentes (aériennes) ou express pour satisfaire les commandes urgentes ... ou bien pour pallier le retard provoqué par notre chaine de production ce qui augmentent évidemment nos coûts logistiques » (Entreprise B, Responsable Approvisionnement).

\section{Verbatims associés aux incertitudes internes à la chaine logistique}

«...Chaque 6 mois nous recevons normalement de la part de nos clients ce qu'on appelle un PDP mais chaque 4 semaines fermes on reçoit toujours des variations de la commande ce qui nous perturbe la planification de la production... ». (Entreprise D, Responsable de planification de la production et JIT).

«.. En raison des perturbations locales ou techniques, on risque de perdre la capacité de notre production ce qui nous risque de ne pas satisfaire les commandes des clients ... Il y a aussi l'émission retardée du plan d'embarquement suite au retard de la chaine de production ou à la déclaration erronée de la production » (Entreprise C, Manager logistique).

\section{Verbatims associés aux incertitudes liées à l'amont de la SC}

«Le manque de fiabilité de certains fournisseurs en matière du respect des quantités, qualité et délai est également une incertitude à gérer pour notre supply chain». (Entreprise A, Coordinateur achat).

«... l'éloignement géographique de nos fournisseurs allonge et complique le circuit logistique... les flux de livraison sont très contraints ; les trajets sont très longs et font intervenir plusieurs opérateurs et modes de transport».(Entreprise F, Responsable Achat).

« ...pour certains composants clés, nous avons le problème des fournisseurs uniques, on reste toujours dépendant des capacités de ces fournisseurs à nous livrer nos commandes ». ». (Entreprise B, Responsable Approvisionnement).

\section{Verbatims associés aux incertitudes liées aux systèmes d'information}


«Le recours à une multitude des applications informatiques pour satisfaire nos besoins en informations rend plus complexe l'accès à l'information recherchée, ce recours s'explique par le fait que notre SI est incapable de gérer l'ensemble de nos tâches et de répondre à nos besoins ce qui rend l'accès à l'information difficile et peut engendrer une distorsion de l'information circulée tout au long de la chaine logistique». (Entreprise C, Manager logistique)

«...il y a le problème de fiabilité des données qui vient d'un manque de formation pertinente du personnel sur les progiciels et les applications utilisées...». (Entreprise D, Planificateur)

« Les informations disponibles parfois ne reflètent pas la réalité du terrain ce qui nous amène à prendre des mauvaises décisions ». (Entreprise E, Approvisionneur)

«...les informations ne sont pas pertinentes et ne sont pas adaptées à nos besoins... ». (Entreprise G, Operations Manager)

« Il y a toujours le blocage du système et l'information n'est pas actualisée et facilement accessible ». (Entreprise D, Approvisionneur)

« Nous sommes disponibles et réactifs en cas d'un problème technique lié au SI, si le problème dépasse nos capacités, notre service support SI à la centrale intervient mais généralement cela entraine un retard d'intervention qui peut augmenter les risques opérationnels ». (Entreprise D, IT Manger)

\section{Verbatims liés aux incertitudes partenariales}

\section{Verbatims associés aux incertitudes liées à la confiance}

« ...la confiance est étroitement liée au degré de partage et de collaboration des partenaires, nous avons des clients qui partagent leurs prévisions et nous communique à l'avance chaque éventuel changement, donc on se sent sécurisé et très à l'aise de travailler avec. Mais pour d'autres, ce n'est pas le cas, on doit être toujours à l'affût de tout changement ce qui perturbe nos prévisions ». (Entreprise C, Manager logistique).

\section{Verbatims associés aux incertitudes liées à l'engagement}

« Nous avons des contrats de longue durée avec nos partenaires, c'est donc un investissement mutuel en temps et en argent... Notre entreprise s'investit dans la mise en place des cellules d'animation de la performance logistique fournisseurs dans notre usine afin d'améliorer la qualité logistique et la ponctualité des livraisons de ses fournisseurs...». (Entreprise B, Approvisionnement).

\section{Verbatims liés aux incertitudes des tâches}

\section{Verbatim associés aux incertitudes liées la variété des tâches}

« Notre procédure nous décrit les tâches courantes à effectuer, bien sûr cela réduit certains de nos problèmes... Il y a des imprévus ou des cas urgents où on ne trouve pas toujours un repère dans la procédure, et parfois même l'application de la procédure dans ces cas particuliers complexifie encore le travail et entrave une prise de décision efficace ». (Entreprise A, Coordinateur achat).

\section{Verbatim associés aux incertitudes liées à l'interdépendance des tâches}

« Notre service a une étroite relation avec les autres services logistiques, cette interdépendance nous provoque une perte de temps dans l'accomplissement des tâches et même dans l'identification de l'origine des erreurs, surtout qu'il y a un manque de coordination interactive entre certaines fonctions ». (Entreprise C, Planificateur).

\section{Verbatims liés aux capacités du système d'information}

\section{Verbatim associé à la capacité « Capacité du traitement de l'information»}

« Notre SI répond à nos besoins en logistique, si le facteur social est capable de produire l'information exacte et de la transférer au bon moment $»$. (Entreprise C, Manager logistique) 


\section{Verbatim associé à la capacité « Communication et partage d'informations en interne $\gg$ \\ «... Les fonctionnalités du SI nous permettent de communiquer et d'échanger des informations en interne et permettent de nous résoudre des problèmes en collaboration mais à condition que les informations alimentées dans le système soient fiables et de qualité... » (Entreprise C, Manager logistique).}

Verbatim associé à la capacité « Communication et partage d'informations en externe $\gg$

« Grâce à notre communication étroite et des interfaces intégrées avec nos partenaires, nous pouvons échanger, intégrer et traiter les informations en collaboration». (Entreprise C, Approvisionneur)

\section{Verbatim associé à la capacité « Aide à la décision »}

«... notre SI nous permet d'anticiper les ruptures, nous avons des états de gestion de suivi du stock ... il nous permet de débloquer des problèmes et de faciliter la prise de décision au bon moment... ». (Entreprise B, North Africa Cluster Central Freight Coordinator)

\section{Les antécédents de la résilience}

\section{Verbatim associé à la dimension «Intégration»}

« Nous avons 2 sites sur Tanger ayant la même hiérarchie, donc on partage tous, on est séparé juste physiquement. $\mathrm{Au}$ sein de notre entreprise nous avons des systèmes d'information et de communication forts qui nous permettent de communiquer toutes les informations requises et qui nous facilite la collaboration interservices...». (Entreprise D, Responsable de planification de la production et JIT)

« Nous associons nos clients dans les différentes phases des projets en les impliquant dans notre processus de conception des produits. Une fois le projet est validé, nous restons en contact avec les clients via des réunions régulières afin d'anticiper leurs demandes ». (Entreprise C, Manager logistique)

«On est toujours en contact avec nos fournisseurs, ils connaissent la quantité qu'on a en stock et ils ont des prévisions plus ou moins sur notre date de passation des commandes, en cas d'une déviation, nos fournisseurs peuvent réajuster la livraison selon nos besoins grâce à une collaboration réciproque ». (Entreprise D, Approvisionneur)

\section{Verbatim associé à la dimension « Flexibilité »}

« Nous avons des contrats sur système pour chaque fournisseur, on peut modifier les conditions soit à la hausse ou à la baisse en termes de quantités et même le lead time on peut le changer via l'EDI .Donc nous avons des fournisseurs flexibles qui veillent à la satisfaction de nos besoins ». (Entreprise A, Coordinateur achat)

« La plupart de nos matières premières sont utilisées dans nos différents projets, donc en cas d'une rupture elles sont interchangeables ». (Entreprise C, Approvisionneur)

« Pour la plupart des matières, nous avons plusieurs fournisseurs, mais pour certaines, nous avons un seul fournisseur qui détient le monopole, normalement le choix de fournisseurs se fait au niveau de notre centrale qui se charge de leur sélection et jusqu'à maintenant, elle cherche à nous trouver d'autres fournisseurs capables de doubler ce dernier ». (Entreprise E, Manager logistique)

« Nous avons vraiment des collaborateurs très flexibles qui s'adaptent aux changements des spécifications des clients, ils sont prêts à travailler des heures sup et en mode shift pour satisfaire les commandes clients ». (Entreprise F, Manager logistique)

«...on fait recours à n'importe quel mode du transport juste pour arriver à livrer au temps voulu par les clients (Airfreight/Charter,)». (Entreprise E, Responsable Transport et douane) 


\section{La résilience de la chaine logistique}

\section{Verbatim associé à la dimension «Capacité d'anticipation »}

« Nos fournisseurs sont intégrés dans nos processus de production, et même nous avons des équipes destinées à travailler chez les fournisseurs, pour anticiper toute problématique liée à nos commandes ». (Entreprise A, Coordinateur achat)

Verbatim associé à la dimension « Capacité de réponse »

«C'est grâce à notre collaboration interne que le problème a pu être résolu, nous avons ce qu'on appelle « sister plants » qui sont des usines de notre entreprise éparpillées dans le monde. En Ukraine, nous avons aussi notre usine c'est celle qui nous a prêté leur numéro d'enregistrement de TVA afin de débloquer l'opération de dédouanement ». (Entreprise E, Responsable Transport et douane)

\section{Verbatim associé à la dimension « Capacité de rétablissement »}

«Suite à la plupart des perturbations rencontrées, nos processus logistiques reviennent à leur état normal de fonctionnement voir parfois on note certaine amélioration apportée à la façon dont on gère nos opérations logistiques ». (Entreprise A, Approvisionneur)

Verbatim associé à la dimension « Capacité d'apprentissage »

«...on a appris le fait d'avoir toujours un plan B, cette expérience nous a permis d'acquérir des compétences et des capacités cognitives additionnelles à exploiter dans des éventuelles futures perturbations». (Entreprise C, Manager logistique) 